\title{
Forensic engineering of advanced polymeric materials Part IV: Case study of oxo-biodegradable polyethene commercial bag - aging in biotic and abiotic environment
}

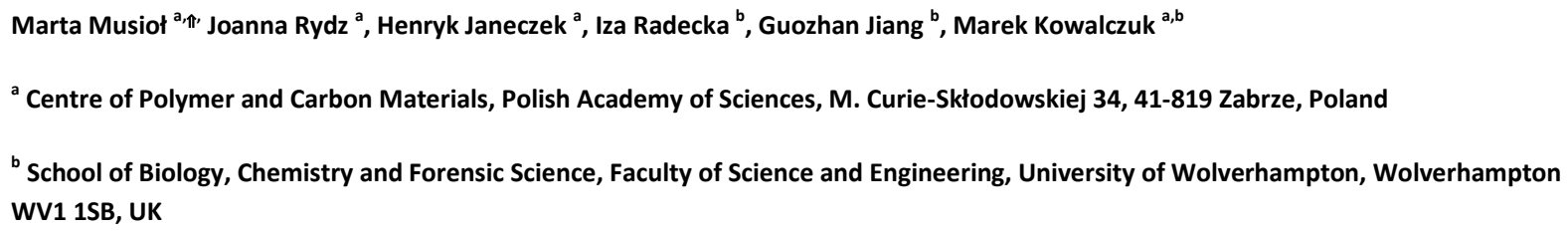

\section{Abstract}

The public awareness of the quality of environment stimulates the endeavor to safe polymeric materials and their degradation products. The aim of the forensic engineering case study presented in this paper is to evaluate the aging process of commercial oxo-degradable polyethylene bag under real industrial composting conditions and in distilled water at $70^{\circ} \mathrm{C}$, for comparison. Partial degradation of the investigated material was monitored by changes in molecular weight, thermal properties and Keto Carbonyl Bond Index and Vinyl Bond Index, which were calculated from the FTIR spectra. The results indicate that such an oxo-degradable product offered in markets degrades slowly under industrial composting conditions. Even fragmentation is slow, and it is dubious that biological mineralization of this material would occur within a year under industrial composting conditions. The slow degradation and fragmentation is most likely due to partially crosslinking after long time of degradation, which results in the limitation of low molecular weight residues for assimilation. The work suggests that these materials should not be labeled as biodegradable, and should be further analyzed in order to avoid the spread of persistent artificial materials in nature.

\section{Introduction}

The use of plastic products, especially polyolefins, has increased considerably in recent years due to their good processability, mechanical properties, durability and low cost (Ammala et al., 2011; Restreopo-Folrez et al.,2014). The fact that conventional plastics do not degrade easily in nature is somewhat problematic since parallel to the growth in use the amount that inadvertently finds its way into nature is increasing as well (Bekyarova et al., 2007; Roy et al., 2005). Difficulties in ensuring efficient management of plastic waste in the municipal solid waste stream require new solutions, particularly for the packaging industry (Musioł et al., 2010; 2011). The "back to nature" via biodegradation seems to be a very attractive approach (Chapman, 2012). Narayan (2016) pointed out that "biodegradation is the processes during which plastics products are completely removed from the environment compartment in a timely, safe and efficacious manner by use of microorganisms existing in the disposal environment".

Oxo-biodegradation is described as complex series of chemical reactions with the action of oxygen, ultraviolet light and/or heat in which the long chains of polyethylene molecules are broken down. The term "oxo-biodegradation" is used when the both degrading phenomenon are demonstrated (Thomas et al., 2010).

Polyethylene is generally considered as a non-biodegradable plastic widely used as a packaging material. For several decades producers of conventional polyethylene are searching for methods 
which would accelerate the degradation of the material in the environment. Solutions include the introduction of "weak" structural elements such as carbonyl groups in the hydrocarbon backbone or side chains, olefinic bonds, the addition of a biodegradable polymer, or the use of pro-oxidants or photoinitiators (Albertsson et al., 1998; Magagula et al., 2009; Martelli et al., 2009; Roy et al., 2008; Rydz et al., 2016). The goal of all these approaches is to develop lifetime programmable polyethylene (Albertsson et al., 1998). Most degradable polyolefin systems are designed to undergo oxidation in air (oxo-degradation). The changes in chemical structure resulting from oxidation should lead to the breakdown of the polymer chain into smaller fragments which can then be bioassimilated and mineralized (Ammala et al., 2011). This is in line with polymer degradation meaning changes in polymer properties by bond scissions and subsequent chemical transformation (Singh and Sharma, 2008).

Currently, a number of polyolefin products are marketed as "oxo-biodegradable" where the degradation is described to consist of a two-stage process. The first is an abiotic process in which oxygen from the air reacts with the polymer which then undergoes chain scission resulting in low molecular fragments with functional groups such as carboxy, formyl hydroxy and ester linkage (Albertsson et al., 1998; Ammala et al., 2011; Chiellini et al., 2006). Products of the oxidation initiated by heat are very similar to those resulting from photo degradation (Wiles and Scott, 2006). The second stage is a biotic process involving the consumption of polymer oxidation products by microorganisms (Ammala et al., 2011).

For a polymer to undergo biodegradation, a reduction of molecular weight has to take place at the first stage. Only after that bioassimilation, can the second step occur. It is not very clear how much degradation is needed before the microorganisms are able to attack it for bioassimilation. Haines and Alexander (1974) reported that only alkanes with low molecular weights ( $<620 \mathrm{Da}$ ) can be biodegraded. Restreopo-Folrez et al. (2014) reported the molecular weight should be less than 50 carbons. Yoon et al. (2012) investigated the biodegradation of low molecular weight polyethylene (LMWPE). They stated that the PE with molecular weight up to 1700 in presence of Pseudomonas sp. E4 underwent biodegradation reaching at almost $30 \%$ after 80 days. Generally speaking, the molecular weight should be much smaller than the virgin plastics. This means that a drastic reduction of the polyolefin molecular weight must occur as a condition for later biotic mineralization. Also, a significant disintegration of the material was observed before the molecular mass reduced to 5000 (Albertsson et al., 1998). The fate of the fragmented material is a great concern because they may undergo other changes like crosslinking, resulting in persistence in the environment (Thomas et al., 2012).

The European Commission is still waiting for assessment and legislative conclusion concerning the influence of usage "oxo-biodegradable" plastic bags on the environment. Taking this environmentally responsible decision takes time and detailed research. The packages labeled as "biodegradable" cause a confusion of the consumers. Also, the phrase "oxo-biodegradable" is not clear for the public. Those two words may suggest that people can throw these packages wherever they want, because the microorganisms and oxygen will do the rest. According to the above statements, these materials may be placed in the environment without any control. Thus, determination of the extent of degradation of those kinds of materials in the environment and placing of detailed information on the packages is an urgent task. 
The forensic engineering studies on advanced polymeric materials (FEAPM) deal with the evaluation of the relationships between their structure, properties and behaviour before, during and after practical applications (FEAPM I-III) (Musioł et al., 2016; Rydz et al., 2015; Sikorska et al., 2014). Hauling together specific characterization and optimization of the material properties, its preparation, processing and recycling under the common thread of FEAPM provides a central driving force for the otherwise disconnected works and should allow to avoid failures of the commercial products manufactured from them. In this contribution we describe the FEAPM case study on aging of commercial oxo-biodegradable polyethylene bag (ECObag) under industrial composting conditions and in distilled water at $70^{\circ} \mathrm{C}$.

\section{Experimental}

\subsection{Materials}

The test material was a commercial polyethylene shopping bag ECObag (Figure 1), which was purchased from a Polish supermarket. The bag contained commercial Totally Degradable Plastics Additives (TDPA $\left.{ }^{\circledast}\right)$ and was labeled as an "ECObag - bag that undergoes $100 \%$ biodegradation in 12 months". The thickness of the HDPE film was $22 \mu \mathrm{m}$.

Figure 1. A photograph of the commercial bag ECObag from a Polish supermarket.

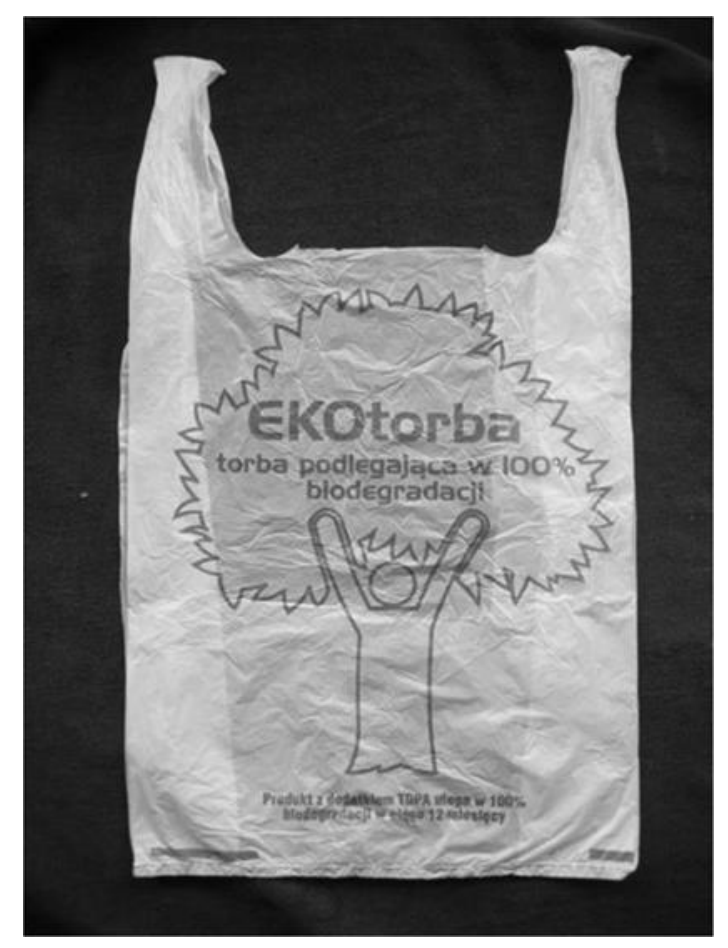

The TDPA ${ }^{\circledR}$ additive are produced by EPI Environmental Technologies Inc., Canada. Normally “oxobiodegradable" bags contain $2 \%$ of TDPA. According to the website of EPI Environmental Products Inc., the level of TDPA ${ }^{\circledR}$ amount is recommended to be in the range of $2-10 \%$ depending on the specific application and the performance required (EPI, 2016).

\subsection{Degradation under industrial composting conditions/in compost environment}


The investigated material consisting of PE and the TDPA ${ }^{\circledR}$ additive was subjected to degradation testing in the composting pile (sample designated as PE-CP), at the Sorting and Composting Station in Zabrze (Upper Silesia, Poland). The compost contained $40 \%$ leaves, $30 \%$ branches and $30 \%$ grass. The static composting open-air pile is located at: latitude $50^{\circ} 18^{\prime} 30.71^{\prime \prime} \mathrm{N}$ and longitude $18^{\circ} 48^{\prime}$ $18.52^{\prime \prime}$ E. Experiments were conducted between late summer and beginning of autumn with daily rainfall below $2.0 \mathrm{~mm}$. The average open-air temperature during this time remained about $12{ }^{\circ} \mathrm{C}$. The compost was not turned during this period. The composting pile was built over a concrete slab with holes to create a chimney effect of airflow. At the time of starting the PE degradation test the composting was already going on for 3 weeks.

A series of 8 specimens (size $4 \times 5 \mathrm{~cm}$ ) was cut from the original bag. The specimens were weighted and inserted into a special stainless steel cage and cover with compost from composting pile (Musioł et al., 2011).

The cage were placed into the composting pile, at the depth of one meter under the surface (total height of composting pile was $4 \mathrm{~m}$ ). The average temperature at the environment (pile) was $64{ }^{\circ} \mathrm{C}$. The average $\mathrm{pH}$ in the composting pile was $\mathrm{pH}=6.9$. During the 10 week test period the variation in pH was below $20 \%(6.3-7.5)$.

After 42 and 70 days of incubation, samples were cleaned by washing in distilled water, drained on blotting paper and then dried to a constant mass in a desiccator held under vacuum at room temperature.

The progress of the PE samples degradation under industrial composting conditions in the pile was estimated by macroscopic and microscopic observations of the polymer surface and by molecular weight changes in the samples after 42 days of incubation.

\subsubsection{Hydrolytic aging in the abiotic laboratory conditions}

\section{Experiment 1}

The investigated material was incubated in distilled water at $70{ }^{\circ} \mathrm{C}$ over a period of 70 days (sample designated as PE-H). The temperature was chosen so as to meet the requirements of accelerated degradation test included in international standard [ISO 15814]. A series of 21 samples measuring approximately $35 \mathrm{~mm} \times 30 \mathrm{~mm}$, were cut from original bag. The samples were inserted into $30 \mathrm{ml}$ screw-capped vials, each containing $25 \mathrm{ml}$ of distilled water, and placed in a laboratory oven at $70{ }^{\circ} \mathrm{C}$. After a specified period, the samples were removed from the vials and dried to a constant mass on blotting paper at room temperature. The progress of PE hydrolysis at $70{ }^{\circ} \mathrm{C}$ was estimated by sample mass loss, macroscopic and microscopic observations of the polymer surfaces.

\section{Experiment 2}

One whole ECObag was put into a screw glass container with $500 \mathrm{ml}$ distilled water and left for 365 days at $70^{\circ} \mathrm{C}$. There was no exposure to light. Molecular weight changes of the samples were estimated after 365 days of incubation.

\subsection{Characterization methods of PE samples before and after degradation.}

\subsubsection{Mass loss}


The mass of the degraded samples was determined using an electronic balance RADWAG WAS 160/X with a precision of $0.1 \mathrm{mg}$. The results for clean and dried samples after incubation in distilled water were compared with those of the respective samples before degradation. The percentage mass loss was determined using equation (1):

$\%$ Mass loss $=\left(\left(m_{o}-m_{t}\right)\right) / m_{o} \times 100$

were $m_{o}$ is the initial mass of the sample, and $m_{t}$ is the mass after determined time of degradation.

\subsubsection{Macro- and microscopic observation of PE samples surface changes}

Changes on the sample surface were visualized using a digital camera Nikon Coolpix L4 (macro photography). Microscopic observation of the PE materials surface, before and after degradation was done by means of Zeiss optical polarizing microscope (Opton-Axioplan) equipped with a Nikon Coolpix 4500 color digital camera. Microscopic observations were performed at a magnification of 120x.

\subsubsection{Molecular weight determination}

The molecular weight and molecular-weight distribution of the PE samples were determined by gel permeation chromatography (GPC, Waters 150-CV) apparatus equipped with two columns: HT3, HT6E, at $135{ }^{\circ} \mathrm{C} .1,2,4-$ Trichlorobenzene stabilized with Santonox-R was employed as a solvent at a flow rate of $1.0 \mathrm{~mL} / \mathrm{min}$. The data was analyzed using polystyrene calibration curves (Albertsson et al., 1998; Pietruszka et al., 2012). The recorded results make the average of at least two measurements. Mass average molecular weight $M_{w}$, number average molecular weight $M_{n}$ and molecular-weight dispersity $\left(M_{w} / M_{n}\right)$ of the PE samples were determined.

\subsubsection{Thermal properties}

\subsubsection{Thermogravimetric analyzis (TGA)}

Thermal characterization was carried out on a Mettler-Toledo TGA/DSC STARe System, from 25 to $800{ }^{\circ} \mathrm{C}$ at a heating rate of $10^{\circ} \mathrm{C} \mathrm{min}^{-1}$, under constant flow of $60 \mathrm{~mL} / \mathrm{min}$ of nitrogen. The TGA data were analysed using the Mettler-Toledo Star System SW 9.30.

\subsubsection{Differential scanning calorimetry (DSC)}

Thermal characteristics of the materials were obtained using the TA DSC 2010 apparatus (TA Instruments, New Castle, DE) at a heating rate of $20{ }^{\circ} \mathrm{C} / \mathrm{min}$, in range of $-80-+200{ }^{\circ} \mathrm{C}$, under the nitrogen atmosphere (flow rate $=50 \mathrm{~mL} / \mathrm{min}$ ). For each sample only one heating scan was performed to evidence the crystallinity of the sample. The instrument was calibrated with indium and gallium standards. The melting temperature $\left(T_{m}\right)$ was taken as the peak temperature maximum of melting endotherm, and the glass transition temperature $\left(T_{g}\right)$ was taken as the midpoint of the increase of the specific heat associated with the transition.

The degree of crystallinity of the samples was determined by calculating the ratio of heat of fusion $\left(\Delta H_{f}\right)$ of sample and heat of fusion of fully crystalline HDPE, $293 \mathrm{~J} / \mathrm{g}$ (Lei et al., 2007; Liang et al., 2004) according to Equation (2):

$X=\Delta H_{f s} / \Delta H_{f c}$ 
where $X$ is the degree of crystallinity, $\Delta H_{f s}$ is the heat of fusion of samples, and $\Delta H_{f c}$ is the heat of fusion of fully crystalline sample.

\subsubsection{Fourier transform infrared spectroscopy (FTIR)}

Changes in the polyethylene structure and the surface of the material, were analyzed by Fourier transform infrared (FTIR) using a Shimadzu IRAffinity-1 Spectrophotometer with MIRacle Attenuated Total Reflectance (ATR) Attachment. Each film sample was scanned 50 times at a resolution setting of $4 \mathrm{~cm}^{-1}$ and averaged to obtain the spectrum. All spectra were scanned over the frequency range of 600 to $4500 \mathrm{~cm}^{-1}$. Triplicate measurements were made on each side of the sample. The relative absorbance intensities of the keto carbonyl bond at $1717 \mathrm{~cm}^{-1}$ (chain end and backbone ketones) and the vinyl bond (double bond, $-\mathrm{C}=\mathrm{C}-$ ) at $1653 \mathrm{~cm}^{-1}$ to that of the methylene bond at $1462 \mathrm{~cm}^{-1}$ $\left(\mathrm{CH}_{2}\right.$ scissoring vibration) were evaluated as keto carbonyl bond index (KCBI) and vinyl bond index (VBI). They are calculated using Equation (3) and (4) respectively:

$$
\begin{aligned}
& \mathrm{KCBI}=I_{1717} / I_{1462} \\
& \mathrm{VBI}=I_{1653} / I_{1462}
\end{aligned}
$$

where $I_{1717}$ and $I_{1462}, I_{1653}$ are the absorbance at $1717,1462,1653 \mathrm{~cm}^{-1}$ respectively.

\section{Results and discussion}

Determination of the degree of degradation of the materials by use of different techniques gives a clear picture of the progress of the process. Evaluation of the changes in polymer molecular weight is important because it is a crucial parameter for many physical properties of the material.

Differences in the thermal stability of the samples during degradation are evaluated by the TGA. The ATR-FTIR spectroscopy determines the changes in functional groups. Using of those techniques in the forensic engineering studies on advanced polymeric materials can help to receive the solution in the development of new materials for specific application.

\subsection{Mass loss}

Within 70 days the mass loss in all samples in all environments remained below one mass percent. No disintegration occurred during this period of time.

\subsection{Visual inspection}

Surface changes were monitored during the experiments performed. Macro- and microscopic observation after 70 days incubation in both environments revealed no surface changes in the samples. Visual changes were observed (Figures.2 and 3) after 365 days of hydrolytic aging.

The disintegration of the material was observed in Experiment 2 for the PE-H sample after hydrolytic aging which was conducted for 365 days in distilled water at $70{ }^{\circ} \mathrm{C}$. After degradation, it was observed that the investigated material is separated into two fractions, large pieces of the investigated material (PE-HL) and small pieces separated from ECObag (PE-HS). Both fractions were further analyzed. 
Figure 2. Digital photographs of PE-H after 365 days of incubation in distilled water at $70{ }^{\circ} \mathrm{C}$ (two fractions: $\mathrm{PE}-\mathrm{HL}$ - large pieces of investigated material, $\mathrm{PE}-\mathrm{HS}$ - small pieces of investigated material).

\section{PE-HL \\ PE-HS}

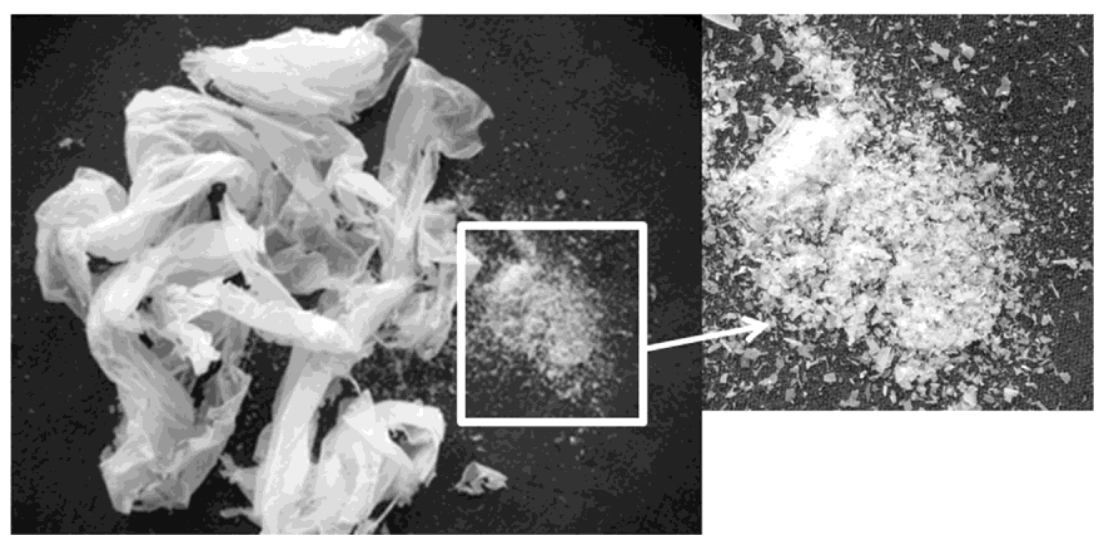

Figure 3. Photomicrographs (120x) of the PE sample surfaces before $(0)$ and after a specific degradation time in selected environments: industrial composting pile (CP) and distilled water at $70{ }^{\circ} \mathrm{C}(\mathrm{H})$ (two fractions: $\mathrm{PE}-\mathrm{HL}$ - large pieces of investigated material, sample PE-HS - small pieces of investigated material).
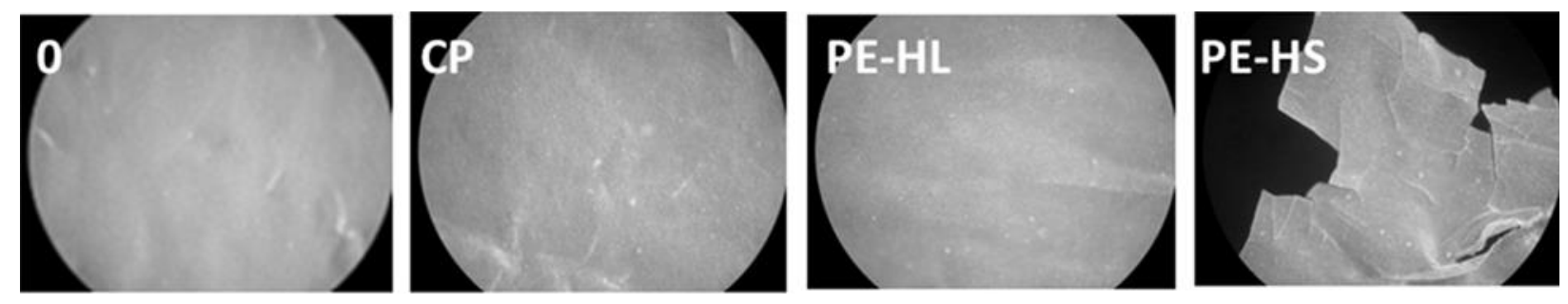

\subsection{Molecular weight changes}

Changes in weight average molecular weight $M_{w}$, number average molecular weight $M_{n}$ and molecular weight dispersity are excellent indicators of the structural changes of the polymer chains (Pietruszka et al., 2012; Reddy et al., 2008). $M_{n}$ is used for the analysis because it reflects the formation of the low molecular weight species (Portillo et al. 2016). Prior to the tests the material had an average molecular weight $M_{n}=29914 \mathrm{~g} / \mathrm{mol}$ and molecular-weight dispersity $M_{w} / M_{n}=11.7$. Average molecular weights and the molecular weight dispersity of the analyzed samples after degradation experiment are listed in Table 1 . The changes in $M_{n}$ are significant. The $M_{n}$ decrease $33 \%$ after 42 days in compost pile. The degradation in distilled water for 365 days was quite similar, with a $30 \%$ reduction for large pieces, and a $47 \%$ reduction for small pieces. According to the results of studies of microbial degradation of aliphatic paraffin only alkanes containing a maximum of 44 carbon atoms (molecular weight $<620 \mathrm{Da}$ ) can be mineralized by microorganisms (Haines and Alexander, 1974). The investigated material after hydrolytic aging for 365 days, still had a molecular 
weight significantly above that level. The molecular weight dispersity of the tested samples is considerably higher after degradation under either of the used conditions, as shown in Table 1.

Table 1. Changes in the dispersity ( $\mathrm{Mw} / \mathrm{Mn}$ ) of the sample during the experiment. Sample $\mathrm{CP}-$ after 42 days degradation in the composting pile, sample $\mathrm{HL}$ - large pieces after hydrolysis at $70^{\circ} \mathrm{C}$; sample $\mathrm{HS}$ - small pieces after hydrolysis at $70^{\circ} \mathrm{C}$.

\begin{tabular}{lllll}
\hline Time [days] & Environment/Sample & $M_{n}[\mathrm{~g} / \mathrm{mol}]$ & $M_{n}$ remaining $[\%]$ & $M_{w} / M_{n}$ \\
\hline 0 & - & 30000 & 100 & 11.7 \\
42 & CP & 20000 & 67.3 & 16.7 \\
365 & HL & 21000 & 70.7 & 16.5 \\
365 & HS & 16000 & 53.0 & 17.4 \\
\hline
\end{tabular}

\subsection{Thermal behavior of Ecobag during degradation}

The decomposition temperature $\left(T_{d}\right)$ and the residue of the PE samples with degradation time are summarized in Table 2 . The decomposition temperature $\left(T_{d}\right)$ was defined as the temperature at $5 \%$ mass loss and the remaining mass was measured at $600{ }^{\circ} \mathrm{C}\left(R_{600}\right)$ (Martelli et al., 2009).

Table 2. TG data of ECObag as a function of degradation time (sample $\mathrm{CP}$ - composting time, sample $\mathrm{H}-\mathrm{hydrolysis}$ at $70{ }^{\circ} \mathrm{C}$, $\mathrm{L}-$ large pieces, $\mathrm{S}$ - small pieces).

\begin{tabular}{lllll}
\hline Time [days] & 0 & $70(\mathrm{CP})$ & $70(\mathrm{H})$ & $365(\mathrm{HL})$ \\
\hline $\mathrm{T}_{d}\left[{ }^{\circ} \mathrm{C}\right]$ & 204 & 325 & 216 & 416 \\
$\mathrm{R}_{600}[\%]$ & 18.6 & 8.9 & 7.3 & 304 \\
\hline
\end{tabular}

$\mathrm{T}_{d}$ is a decomposition temperature defined as $5 \mathrm{wt} \%$ weight loss, $\mathrm{R}_{600}$ is the remaining mass at $600{ }^{\circ} \mathrm{C}$, time 0 corresponds to the sample before degradation.

It can be seen from Table 2 that there is an increase in $T_{d}$ and a decrease in residue mass after degradation in both the composting conditions and the distilled water conditions. The increase in $T_{d}$ after degradation could be attributed to an increase in the amount of more stable material that degrades at a higher temperature. This result could imply the formation of cross-link, which lead to higher thermal stability (Martelli et al., 2009). The decrease in residue mass could be attributed to the leaching of the low molecular weight fragments or commercial additives during incubation (Roy et al., 2008).

After one year of hydrolytic aging (sample $365 \mathrm{HL}$ ), the $T_{d}$ of the sample increased from $204{ }^{\circ} \mathrm{C}$ to $416{ }^{\circ} \mathrm{C}$, and for the small pieces ( $365 \mathrm{HS}$ ) increased from $204{ }^{\circ} \mathrm{C}$ to $304{ }^{\circ} \mathrm{C}$. However, the residual mass was higher for the small pieces (15.2\%) than for the large pieces (12.4\%). These results may suggest the presence of low molecular weight chains in the small pieces (Musiot et al., 2010). This is consistent with the molecular weight measurements. The discrepancy in the residual mass could be due to the presence of impurities (commercial additives) in the material or the crosslinking of the investigated material. The presence of crosslinking can be further confirmed in the following dissolution test. Crosslinking of oxo-degradable polyethylene mulch film was also observed by Feuilloley et al. (2005). 
The first derivative TG (DTG) traces of the samples shown in Figure 4 are for hydrolytic degradation at $70^{\circ} \mathrm{C}$ for 70 days (2), and 70 days' composting (3) in comparison with the sample before degradation (1). Figure 5 shows the DTG traces of three samples before degradation (1), big pieces after 365 days' hydrolytic degradation (2) and small pieces after 365 days' hydrolytic degradation (3). It can be seen that the decomposition peak temperature $T_{\max }$ increased compared with starting material.

Figure 4. First differential TGA curve of ECObag, 1 - before degradation, 2 - after 70 days degradation in distilled water at $70^{\circ} \mathrm{C}$ and $3-$ after 70 days degradation in composting pile.

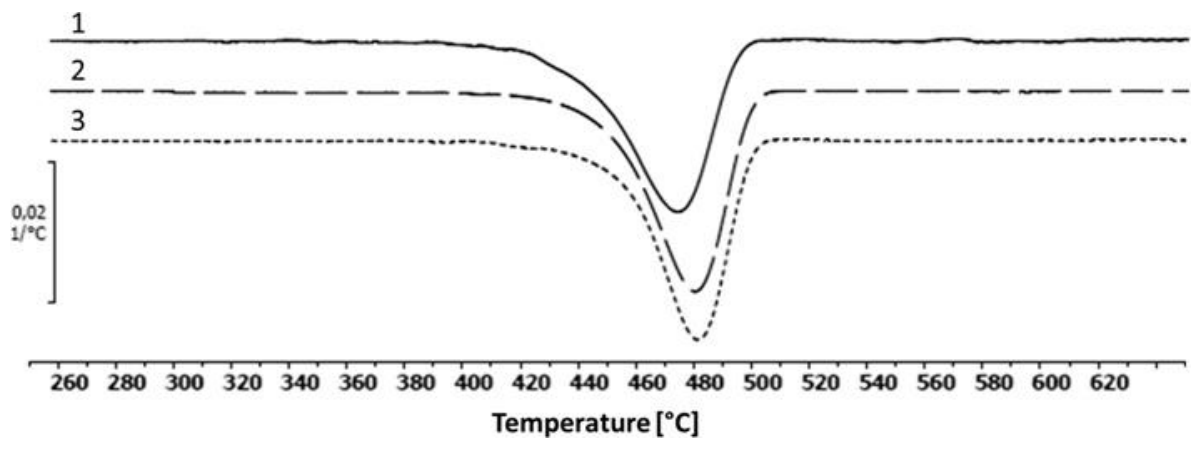

Figure 5. First differential TGA curve of ECObag, 1 - before degradation, 2 - after 365 days degradation in distilled water at $70^{\circ} \mathrm{C}$ from the large piece and $3-$ after 365 days degradation in distilled water at $70^{\circ} \mathrm{C}$ from small pieces.

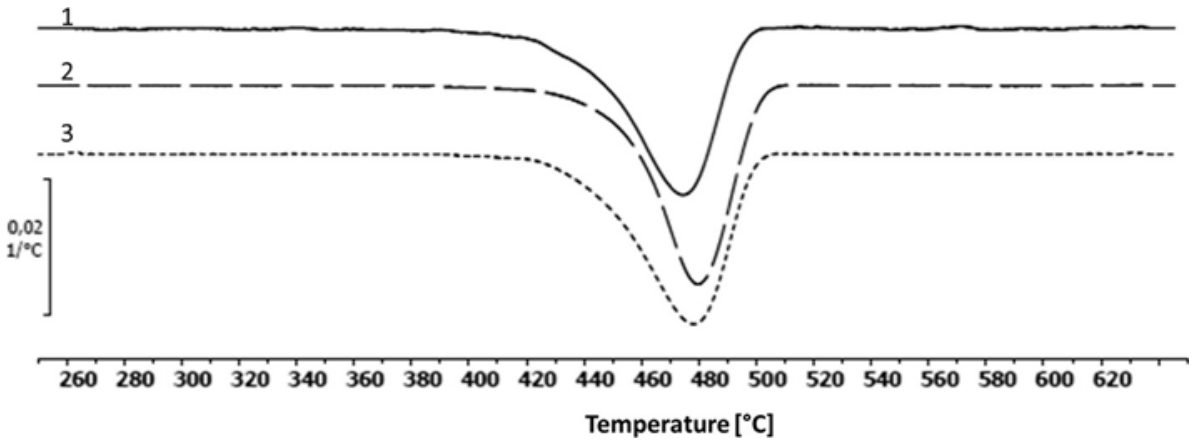

In comparison with the large pieces, lower $T_{d}$ and molecular weight for the small piece sample (HS) does not correspond to a lower $\mathrm{T}_{\max }$, which is quite similar to that of the large piece sample. During aging of PE in different environment branching of the material may be expected. However, this phenomenon would cause a decrease of thermal stability (Beyler and Hirschler, 1995). In our case, partial crosslinking of low molecular weight fragments is probably responsible for the similar $T_{p}$.

The melting point, glass transition temperature and crystallinity of the samples were analyzed using differential scanning calorimetry (DSC). The DSC scans are plotted in Figure 6 for the samples before degradation (a), big pieces after 365 days' hydrolytic degradation (b) and small pieces after 365 days' hydrolytic degradation. The melting temperature of the PE samples had a slight decrease after 365 days of degradation. The small piece sample after 365 days of incubation in distilled water at $70{ }^{\circ} \mathrm{C}$ had a lower $T_{m}$ than that of the large piece sample. The lower $T_{m}$ could be due to the lower 
molecular weight (see GPC data). Colom et al. (2003) reported that the decrease of the decomposition and melting temperatures is associated with shorter polymeric chains and a lower thermal stability of the material.

The crystallinity of the PE samples after 365 days of degradation increased from 62 to $70 \%$ for the large pieces and decreased to $37 \%$ for the small pieces. The starting PE sample had an initial crystallinity index of $62 \%$, and the amorphous phase allows absorbing oxygen. It was found that thermo-oxidative degradation occurs with the presence of oxygen which is absorbed by the amorphous region of polyethylene (Lampman, 2003). The increase in crystallinity for the PE samples is probably due to the chain scission along the amorphous regions. The chain scission allows the freed segments to crystallize (Khabbaz et al., 1999). The creation of new intermolecular polar bonds, due to carbonyl groups may also lead to this effect (Khabbaz et al., 1999; Sebaa et al., 1992).

It is apparent from the DSC traces of the starting PE and the PE after degradation that the peak melting point is well defined for starting PE, less defined for the large pieces of PE after 365 days of degradation and even less defined for the small pieces PE after 365 days of degradation. This is due to the crystallinity of the material. The starting PE and the large pieces of PE after 365 days had less branching and cross-linking than small pieces of PE. This results in a more crystalline solid, which in turn results in a sharper peak melting point. This is consistent with what was observed in the GPC analysis which showed that the PE-HL undergoes less degradation than PE-HS under investigated conditions.

Figure 6. DSC plot of PE samples, a - before degradation, b-after 365 days incubation in distilled water at $70^{\circ} \mathrm{C}$ of large piece and cafter 365 days incubation in distilled water at $70^{\circ} \mathrm{C}$ of small pieces.

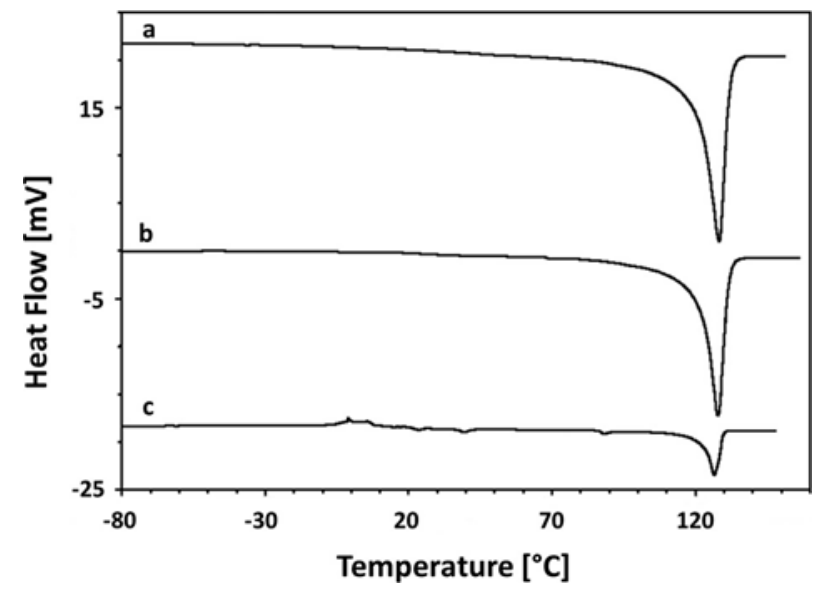

In the study of PE degradation in sea water, Jha et al. (2013) observed a decrease in the crystallinity with 30 days incubation. We found a slight increase in the crystallinity of the PE during degradation experiments. The degree of crystallinity of the large piece (PE-HL and PE-CP) increased from $62 \%$ to around $70 \%$ after 365 days in distilled water, and the degree of crystallinity of the composted sample increased from $62 \%$ to $66 \%$. For the small piece sample (PE-HS) after 365 days incubation in distilled water at $70{ }^{\circ} \mathrm{C}$, a significant decrease of the degree of crystallinity was observed from $62 \%$ to $37 \%$. This may suggest the partially crosslinking of the sample during the degradation testing. 
Increase in crosslinking causes the reduction of polymer chains mobility and hence limitation in arranged into lamellae fold and decrease in crystallinity (Tamboli et al., 2004).

\subsection{Dissolution test}

The above results indicate that the material crosslinked after degradation. Crosslinked PE is insoluble and the polymer chains joined together in a three-dimensional network (Tamboli et al., 2004). Hence the dissolution test of the samples was conducted at $135^{\circ} \mathrm{C}$ in 1,2,4-trichlorobenzene to confirm the presence of crosslinking. The small piece sample after 365 days incubation in distilled water at $70{ }^{\circ} \mathrm{C}$ was almost completely dissolved. However, a small amount of material remained, which suggests the presence of crosslinked material after degradation.

\subsection{FTIR analysis}

Hydrophobic polyethylene without hydrolysable bonds is expected to be a stable polymer. However, enzymes are able to catalyze the oxidation, reduction, hydrolysis and esterification reactions as well as molecular transformations. Therefore, it is possible the microorganisms can degrade PE (Restreopo-Folrez et al., 2014).

Figure 7. FTIR spectra of biotic (a) and abiotic (b) degradation of polyethylene samples.

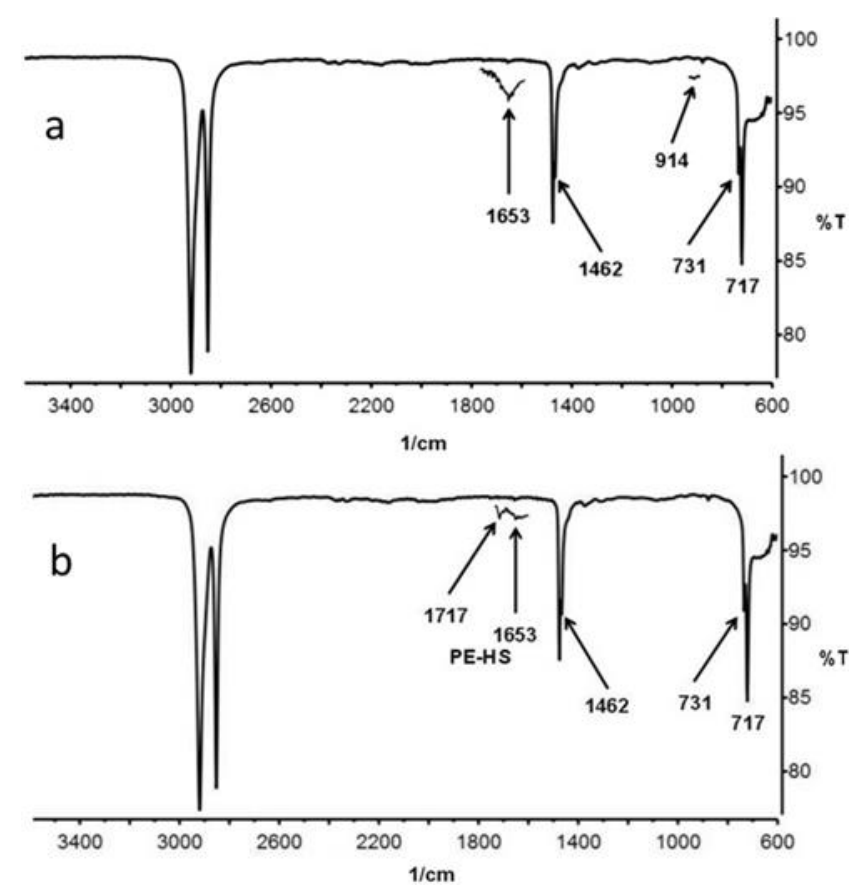

The spectra of the PE samples are shown in Figure 7. The main bands belongs to $\mathrm{C}-\mathrm{H}$ stretching asymmetric and symmetric vibrations at 2914 and $2849 \mathrm{~cm}^{-1}, \mathrm{CH}_{2}$ scissoring vibrations at 1472 and $1462 \mathrm{~cm}^{-1}$ as well as, $\mathrm{CH}_{2}$ rocking vibrations at 731 and $717 \mathrm{~cm}^{-1}$. Keto Carbonyl Bond Index (KCBI) and Vinyl Bond Index (VBI) were calculated from the FTIR spectra using Equations 3 and 4 . The two indices were used as parameters to monitor the degree of degradation of the polyethylene films in industrial composting pile (biotic degradation) and distilled water (abiotic degradation). The calculated indices were plotted in Figure 8. The data obtained from the FTIR spectra of the examined films confirm an increase in carbonyl as well as double bond index during degradation in both 
environmental conditions: the industrial composting pile and distilled water. The increase in the double bond in the industrial composting pile is in accordance with the mechanism proposed by Albertsson et al. (1998), who described the formation of double bonds as a result of biodegradation. The results of this study demonstrate that a slight increase in double bonds is observed not only during exposure to biotic environment but also in hydrolytic degradation. An increase in Keto Carbonyl Bond Index occurred mainly during degradation in the abiotic environment as described previously.

Figure 8. FTIR analysis of plain PE, PE incubated in distilled water (large - HL and small - HS pieces) and PE incubated in industrial composting pile (PE-CP); KCBI - Keto Carbonyl Bond Index; VBI - Vinyl Bond Index. The standard error bars indicated.

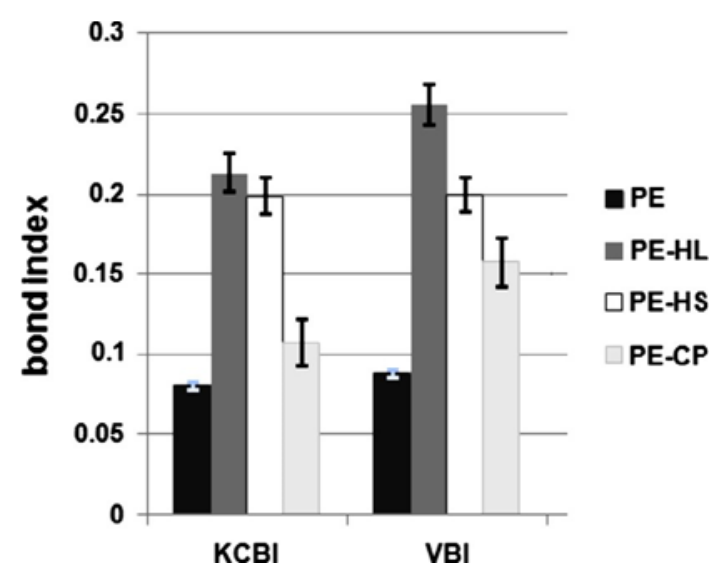

We did not observe any oxygenated functional groups in the FTIR spectra, which may be due to the lack of microorganisms in contrast to the sea water environment. However, we did observe a decrease in molecular weight and an increase in the bond indices, which is most probably caused by the oxidative additives in the PE. The oxidative processes cleaved the polymer chains, leading to easier mobility of the chains and thus an increase in the crystallinity. The smaller double bond indices in the small piece samples may suggest that the double bonds were used for crosslinking.

\section{Conclusions}

In the present study a commercial oxo-degradable polyethylene commercial bag was evaluated during aging in biotic and abiotic environment. Samples exposed to industrial composting and to hydrolytic conditions for up to 70 days have been found to undergo only modest change.

The hydrolytic aging was extended to 365 days. After this period same changes in the investigated material were observed. The disintegration of the samples allowed for isolation of the large and small pieces of the material which were further analyzed. The maximum reduction in average molecular weight was $47 \%$ in the small parts of the samples.

Thermal data confirms the minor changes seen from molecular weight measurements. With progressive degradation the temperature of maximum degradation rises. It was found that PE generally degraded to a lower molecular weight, however PEHL tended to produce a more crystalline product which showed a better defined peak melting than PE-HS. 
The results indicate that part of the PE after aging in the environment may crosslink leading to remaining in the environment which is difficult for further degradation. Although the bag studied was from Polish market, the products containing TDPA ${ }^{\circledR}$ are expected to have similar composition and degradation performance.

The conclusion of these experiments is that oxo-degradable products offered on the markets degrade very slowly under composting conditions. Even fragmentation is slow. Furthermore, there is no possibility that biological mineralization would occur on these materials within a year. Our results show that these materials should not be labeled as biodegradable, with the understanding of ISO 13432 , and should be further analyzed in order to avoid the spread of persistent artificial materials in nature.

Hoverer, PE wastes could be valuable materials for production compostable polymers as e.g. reported recently by some of us on the ability of bacteria to produce biodegradable polyhydroxyalkanoates (PHA) using oxidized polyethylene wax as a novel carbon source (Radecka et al. 2016).

\section{Acknowledgements}

This work was supported by FP-7 project BIOCLEAN contract No. FP7 - KBBE.2012.3.5-02 and by the European Commission under the Seventh Framework Programme (POLINNOVA project no. 316086).

This work was supported by the National Science Centre, Poland (NCN SONATA 11 project no. 2016/21/D/ST8/01993, "Multifaceted studies on the (bio)degradability profile of composites of selected biodegradable polymers with natural fillers and bacteriocins").

The outcomes of this research will be used to implement the PLASTiCE project (3CE368P1, "Innovation value chain development for sustainable plastics in Central Europe", Central Europe Programme, co-financed by ERDF).

\section{References}

Albertsson, A.C., Erlandsson, B., Hakkarainen, M., Karlsson, S., 1998. Molecular weight changes and polymeric matrix changes correlated with the formation of degradation products in biodegraded polyethylene. Journal of Environmental Polymer Degradation 6, 187-195.

Ammala, A., Bateman, S., Dean, K., Petinakis, E., Sangwan, P., Wong, S., Yuan, Q., Patrick, C., Leong, K.H., 2011. An overview of degradable and biodegradable polyolefins. Progress in Polymer Science 36, 1015-1049.

Bekyarova, E., Thostenson, E.T., Yu, A., Kim, H., Gao, J., Tang, J., Hahn, H.T., Chou, T.-W., Itkis, M.E., Haddon, R.C., 2007. Multiscale carbon nanotube-carbon fibre reinforcement for advanced poxy composites. Langmuir 23, 3970-3974.

Beyler, C.L., Hirschler, M.M., 1995. Thermal decomposition of polymers, in: Beyler, C.L., Custer, R.L.P., Walton, W.D., Watts, J.M.J., Drysdale, D., Hall, J.R.J., Dinenno, P.J. (Eds.), SFPE Handbook of Fire Protection Engineering 2nd ed. National Fire Protection Association, Quincy, Massachusetts, USA, pp. 1-111 - 111-131. 
Bioplastics., E., 2015. "Oxo-biodegradable" plastics and other plastics with additives for degradation. $<$ http://docs.european-bioplastics.org/2016/publications/bp/EUBP_bp_additivemediated_plastics.pdf> (accessed 25th August 2016)

Chapman, G.M., 2012. Back to nature. <http://www.add-xbiotech.com/pdf/P1417_Materials_PKG_Mar-April_12.pdf> (accessed 25th August 2016)

Chiellini, E., Corti, A., D'Antone, S., Baciu, R., 2006. Oxo-biodegradable carbon backbone polymers Oxidative degradation of polyethylene under accelerated test conditions. Polymer Degradation and Stability 91, 2739-2747.

Colom, X., Canavate, J., Sunol, J.J., Pages, P., Saurina, J., Carrasco, F., 2003. Natural and artificial aging of polypropylene-polyethylene copolymers. Journal of Applied Polymer Science 87, 1685-1692.

EN 13432:2000: Packaging. Requirements for packaging recoverable through composting and biodegradation. Test scheme and evaluation criteria for the final acceptance of packaging

EPI, 2016. Oxo-biodegradable Plastic Technology. <http://www.epi-global.com/en/epitechnology.php> (accessed 25th August 2016)

Feuilloley, P., Cesar, G., Benguigui, L., Grohens, Y., Pillin, I., Bewa, H., Lefaux, S., Jamal, M. Degradation of polyethylene designed for agricultural purposes. Journal of Polymers and the Environment 13, 349-355.

Haines, J.R., Alexander, M., 1974. Microbial degradation of high molecular weight alkanes. Applied Microbiology 28, 1084-1085.

Harshvardhan, K., Jha, B., 2013. Biodegradation of low-density polyethylene by marine bacteria from pelagic waters, Arabian Sea, India. Marine Pollution Bulletin 77, 100-106.

Khabbaz, F., Albertsson, A.C., Karlsson, S., 1999. Chemical and morphological changes of environmentally degradable polyethylene films exposed to thermo-oxidation. Polymer Degradation and Stability 63, 127-138.

Lampman, S., 2003. Characterization and Failure Analysis of Plastics. ASM International.

Lei, Y., Wu, Q., Yao, F., Xu, Y., 2007. Preparation and properties of recycled HDPE/natural fiber composites. Composites Part A: Applied Science and Manufacturing 38, 1664-1674.

Liang, G., Xu, J., Bao, S., Xu, W., 2004. Polyethylene/maleic anhydride grafted polyethylene/organicmontmorillonite nanocomposites. I. Preparation, microstructure, and mechanical properties. Journal of Applied Polymer Science 91, 3974-3980.

Magagula, B., Nhlapo, N., Focke, W., 2009. Mn2Al-LDH and Co2Al-LDH-stearate as photodegradants for LDPE film. Polymer Degradation and Stability 94, 947-954.

Martelli, S.M., Fernandes, G.E., Chiellini, E., 2009. Thermal analysis of soil-buried oxo-biodegradable polyethylene based blends. Journal of Thermal Analysis and Calorimetry 97, 853-858.

Musioł, M., Rydz, J., Sikorska, W., Rychter, P., Adamus, G., Kowalczuk, M., 2010. Evaluation of degradation progress of environmental-friendly packaging, 3rd Conference "The Future Of Biodegradable Packaging", Warsaw. 
Musioł, M., Rydz, J., Sikorska, W., Rychter, P., Kowalczuk, M., 2011. A preliminary study of the degradation of selected commercial packaging materials in compost and aqueous environments. Polish Journal of Chemical Technology 13, 55-57.

Musioł, M., Sikorska, W., Adamus, G., Janeczek, H., Richert, J., Malinowski, R., Jiang, G., Kowalczuk, M., 2016. Forensic engineering of advanced polymeric materials. Part III - Biodegradation of thermoformed rigid PLA packaging under industrial composting conditions. Waste Management 52, 69-76.

Narayan, R., 2016. Compostable vs oxo degradable.

<http://www.naturbag.com/pdf/CompostablevsOxoDegradable.pdf> (accessed 25th August 2016)

Pietruszka, A., Bialek, M., Czaja, K., 2012. A Supported Titanium Postmetallocene Catalyst: Effect of Selected Conditions on Ethylene Polymerization. Journal of Applied Polymer Science 123, 1848-1852.

Portillo, F., Yashchuk, O., Hermida, E., 2016. Evaluation of the rate of abiotic and biotic degradation of oxo-degradable polyethylene. Polymer Testing 53, 58-69.

Radecka, I, Irorere, V, Jiang, G, Hill, D, Williams, C, Adamus, G, Kwiecien, M, Marek, A, Zawadiak, Johnston, B, Kowalczuk, M. 2016. Oxidized polyethylene wax as a potential carbon source for PHA production. Materials (MDPI), 9, 367.

Reddy, M.M., Gupta, R.K., Gupta, R.K., Bhattacharya, S.N., Parthasarathy, R., 2008. Abiotic Oxidation Studies of Oxo-biodegradable Polyethylene. Journal of EPolymers and Environment 16, 27-34.

Restreopo-Folrez, J.M., Bassi, A., Thompson, M.R., 2014. Microbial degradation and deterioration of polyethylene - A review. International Biodegradation and Biodeterioration 88, 83-90.

Roy, P.K., Surekha, P., Rajagopal, C., Chatterjee, S.N., Choudhary, V., 2005. Effect of benzil and cobalt stearate on the aging of low-density polyethylene films. Polymer Degradation and Stability 9, 577585.

Roy, P.K., Titus, S., Surekha, P., Tulsi, E., Deshmukh, C., Rajagopal, C., 2008. Degradation of abiotically aged LDPE films containing pro-oxidant by bacterial consortium. Polymer Degradation and Stability 93, 1917-1922.

Rydz, J., Wolna-Stypka, K., Admus, G., Janeczek, H., Musioł, M., Sobota, M., Marcinkowski, A., Krzan, A., Kowalczuk, M., 2015. Forensic engineering of advanced polymeric materials. Part 1 - Degradation studies of polylactide blends with atactic poly[(r,s)-3-hydroxybutyrate] in paraffin. Chemical and Biochemical Engineering Quarterly 29, 247-259.

Rydz, J., Musioł, M., Kowalczuk, M.,2016. Polymers Tailored for Controlled (Bio)degradation through End-group and In-chain Functionalization, Curr. Org. Synth., after review

Sebaa, M., Servens, C., Pouyet, J., 1992. Natural and artificial weathering of low-density polyethylene (LDPE): Calorimetric analysis. Journal of Applied Polymer Science 45, 1049-1053.

Sikorska, W., Adamus, G., Dobrzynski, P., Libera, M., Rychter, P., Krucinska, I., Komisarczyk, A., Cristea, M., Kowalczuk, M., 2014. Forensic engineering of advanced polymeric materials - Part II: The effect of the solvent-free non-woven fabrics formation method on the release rate of lactic and glycolic acids from the tin-free poly(lactide-co-glycolide) nonwovens. Polymer Degradation and Stability $110,518-528$. 
Singh, B., Sharma, N., 2008. Mechanistic implication of plastic degradadion. Polymer Degradation and Stability 93, 561-584.

Tamboli, S.M., Mahaske, S.T., Kale, D.D., 2004. Crosslinked polyethylene. Indian Journal of Chemical Technology 11, 853-864.

Thomas, N.L., Clarke, J., McLauchlin, A.R., Patrick, S.G.,2010. EV0422: Assessing the Environmental Impacts of Oxo-degradable Plastics Across Their Life Cycle. A research report completed for the Department for Environment, Food and Rural Affairs

Thomas, N.L., Clarke, J., McLauchlin, A.R., Patrick, S.G., 2012. Oxo-degradable plastics:degradation, environmental, impact, recycling. Waste and Resource Management 165, 133-140.

Wiles, D.M., Scott, G., 2006. Polyolefins with controlled environmental degradability. Polymer Degradation and Stability 91, 1581-1592.

Yoon, M.G., Jeon, H.J., Kim, M.N., 2012. Biodegradation of polyethylene by the soil bacterium and alkb cloned recombinant cell. Journal of Bioremedation and Biodegradation 3, paper \#145 